\title{
Abstract Design Theory
}

\author{
Yuzuru KAKUDA* and Makoto KIKUCHI**
}

\section{Introduction}

Design is an activity for realizing desire or intention in the objective world. In engineering, the objects which realize our desire are usually physical entities in the real world, like as machines in mechanical engineering, buildings in architecture and so on, and the desire is usually specified as the functions of such substances. We utilize the physical laws which govern the real world in designing physical entities. The functions of an entity are described by its behavior, and the behavior of a physical entity is determined by the attributes of the entity, as well as the physical laws they are governed by. Hence, design is possible as long as we have sufficient knowledge about the relation between the functions and attributes of these physical entities. This is the basic philosophy of Yoshikawa's General Design Theory [7]. His approach was to mathematically express this basic idea by the comparison of the coarseness of two topologies of the functional and attribute spaces. He gave the definition of the functional space in an axiomatic way, but how can we get the functional space?

Function is the concept by which we explain the behavior of entities. This concept inhabits the world of knowledge, while the attribute concept is contained in the real physical world. Design is the activity which connects these two worlds. When we do design, information flows between the world of knowledge and the real world, and we can obtain a functional space from this flow. This is a general scheme which can be found in many examples of the human activity of problemsolving. Programmers produce software satisfying the specifications required by their customers. Specifications are problems to be solved, and software are answers to the problems. The objective world is the set of software, and their knowledge worlds are ruled by the regulations of the actions of software. Programming a software induces a flow of information between a programmer's consciousness and the universe of software. Mathematicians want to find proofs for their conjectures. For this case, the objective world is the collection of proofs, and their minds enjoy

* Department of Computer and Systems Engineering, Kobe University, Kobe 657-8501, Japan. kakuda@kobe-u.ac.jp

** Department of Computer and Systems Engineering, Kobe University, Kobe 657-8501, Japan. mkikuchi@kobe-u.ac.jp 
a logical coherency based on their current mathematical knowledge. Painters depict motifs in their soul by painting in oils. Their heart respects the principles from their tradition, their experience, and their passion. In this case, such principles can be very subjective and even irrational in some cases. In these phenomena, we can find information flows which associate the world of mind with the objective world in the activity of problem-solving.

Abstract Design Theory is a mathematical theory of design which aims to abstract the common features from such problem-solving phenomena based on Channel Theory, a theory of information flow by Barwise and Seligman [1]. Although we are living in a world filled with information flow, it is not easy to find the appropriate flow of information for our desire. A channel is a basic notion of the theory of Barwise and Seligman and it is a principal component of the medium which makes the flow of information. When we are faced with a problem, we choose a plan to solve it. Architects draw blueprints of houses before starting the construction. Programmers write programs in high-level languages, and mathematicians construct outlines of proofs. Painters draw sketches for paintings and composers score music on sheets. All these are examples of channels for the information flow in problem solving. We call these channels in problem solving medium spaces. Finding a suitable medium space for a given problem is an important factor in problem-solving activity. Abstract Design Theory intends to analyze design with functional and medium spaces from the viewpoint of information flow, and the purpose of this paper is to propose the framework of the theory.

In Section 1, we analyze how we classify entities by their functions and we shall start with an example of chimpanzees' breaking nutshells. This is an example of a situation which precedes the activity of design and it will be repeatedly referred to in this paper. Definitions of classifications and state spaces, which are basic notions of Channel Theory, will be given in Section 1. Theories will be defined and discussed in Section 2. We shall introduce the concept of functional schemes in Section 3. A functional scheme is a basic diagram which relates the knowledge world and the objective world. We shall also show how a functional logic, a mathematical structure corresponding to a functional space, can be obtained from a functional scheme in Section 3. In Section 4, we will discuss medium systems which allow for the flow of information in design.

The relationship between Yoshikawa's General Design Theory and Barwise and Seligman's Channel Theory was first developed in Kakuda [2]. A mathematical definition of functions emerging by connections is treated separately in Kakuda [3] within the framework of Abstract Design Theory, and an application of Abstract Design Theory to filter circuit is discussed in Toyoda et al. [6]. 


\section{Glassifications and state spaces}

Let us consider a set of objects in our intuition or thought, and imagine the situation that we want to find functions on the objects under a certain circumstance. When the objects are artifacts we can produce for a purpose, we are expecting them to have the functions we intended. For living organisms, we can find their purposeful behavior called functions, to maintain their lives or species which are recognizable at our knowledge level. In either case, we are classifying the objects by functions when we focus our attention to the objects in our mind, although this classification might be incomplete. In classifying objects by functions and examining them, we acquire knowledge about the objects with respect to their functions. How to do this is the essential problem of design, and we shall formulate and analyze the classifications as mathematical structures. To this end, let us start this section by considering an example of chimpanzees.

Consider a group of chimpanzees under the circumstance that they want to break nutshells with stones and pick up the meat inside them. They have a set of stones. For a nutshell at hand, they may beat it with this stone or that one in order to break the nutshell. This is like an experiment for collecting data in that this stone enables them to break the shells of peanuts but not the shells of walnuts. They are analyzing a stone $a$ to see whether it has a function $\alpha$ which means they can break shells of peanuts with $a$ but does not have a corresponding function $\beta$ for shells of walnuts. That is, they are classifying the set of stones by their functionality.

Let $A$ be the set of stones, $\Sigma$ be the set of functions concerning $A$, and $N$ be the set of stones among $A$ which have been already tested by the group. Then, their data about functions of stones can be represented by the pair $\left\langle\Gamma_{a}, \Delta_{a}\right\rangle$ of disjoint subsets of $\Sigma$ for each $a \in N$ such that $\Gamma_{a}$ is the set of functions $\alpha \in \Sigma$ such that they know $a$ has the function $\alpha$, and $\Delta_{a}$ is the set of functions $\beta \in \Sigma$ such that they know $a$ does not have the function $\beta$. Here, $\Gamma_{a} \cup \Delta_{a}$ is a subset of $\Sigma$ and $\Gamma_{a} \cap \Delta_{a}=\emptyset$. The set $K=\left\{\left\langle\Gamma_{a}, \Delta_{a}\right\rangle \mid a \in N\right\}$ represents chimps' knowledge about breaking nutshells with stones. Suppose that the chimps want to have a stone which has every function in $\Gamma$ but not any function in $\Delta$. They refer to their knowledge so as to judge whether they have a suitable stone for their desire. If some $a \in N$ satisfies the requirement expressed by $\langle\Gamma, \Delta\rangle, \Gamma \cap \Delta, \Gamma \cap \Delta_{a}$ and $\Gamma_{a} \cap \Delta$ must be empty. In other words, no stone in $N$ realizes the requirement $\langle\Gamma, \Delta\rangle$ if $\Gamma \cap \Delta_{a} \neq \emptyset$ or $\Gamma_{a} \cap \Delta$ $\neq \emptyset$ for every $a \in N$ or $\Gamma \cap \Delta \neq \emptyset$. In this way, their knowledge forms a theory $T(K)$ if defined as follows; the requirement expressed by $\langle\Gamma, \Delta\rangle$ is inconsistent with $T(K)$ if and only if $\Gamma \cap \Delta_{a} \neq \emptyset$ or $\Gamma_{a} \cap \Delta \neq \emptyset$ for every $a \in N$ or $\Gamma \cap \Delta \neq \emptyset$.

In the course of time, they are aware that some physical states of stones are essential for their having a fixed function. They feel that the volume, weight, 
solidity and shape of a stone concern the functions they met. Let $\alpha$ be a function they consider. They check the volume, weight, solidity and shape of $a$ for each $a$ $\in N$, and get the ranges of volume, weight, solidity and shapes such that the state of $a$ is in these ranges if $\alpha \in \Gamma_{a}$, and not in these ranges if $\alpha \in \Delta_{a}$. If they cannot find the ranges, they feel that other physical states than volume, weight, solidity and shape may concern the function $\alpha$. When $f(\alpha)$ denotes these ranges and $\operatorname{state}(a)$ denotes the volume, weight, solidity and shape of $a$, then state $(a) \in f(\alpha)$ if $\alpha \in \Gamma_{a}$ and state $(a) \notin f(\alpha)$ if $\alpha \in \Delta_{a}$ for all $a \in N$.

They apply this scheme to any stones even not in $N$. For example, consider the situation that all stones in $N$ are used by another group of chimps. They think it has a possibility that a stone $a$ has a function $\alpha$ if state $(a)$ is in the range $f(\alpha)$ for any function $\alpha$ they met. Taking an arbitrary range of volumes, weight, solidity and shapes for $f(\alpha)$ for any function $\alpha$ they did not meet, define a classification $A$ so that $\operatorname{typ}(A)$ is $\Sigma, \operatorname{tok}(A)$ is $A$ and $a \models_{A} \alpha$ if and only if state $(a)$ $\in f(\alpha) . \quad A$ is the classification of the set $A$ of stones by the functions in $\Sigma$ with their limited knowledge. As a result, $\models_{A}$ does not necessarily express the true classification relation between the stones in $A$ and the functions in $\Sigma$. However, $A$ contains at least the information from their knowledge in the sense that $\alpha \in \Gamma_{a}$ implies $a \models_{A} \alpha$ and $\alpha \in \Delta_{a}$ implies $a k_{A} \alpha$. In other words, there exists no stone in $N$ satisfying the requirement expressed by $\langle\Gamma, \Delta\rangle$ if it is inconsistent with their knowledge theory $T(K)$. Put $g(a)=\langle\{\alpha \in \Sigma \mid$ state $(a) \in f(\alpha)\},\{\alpha \in \Sigma \mid \operatorname{state}(a) \notin$ $f(\alpha)\}>$ for $a \in A$. Both directions, $f$ and $g$, which are opposite to each other, are essential to the construction of classification by functions.

In order to give a mathematical explanation for these circumstances, we shall give the definitions of notions in this section. These notions mostly come from Barwise and Seligman [1].

Definition 1.1. A classification $A$ consists of the following things :

1. A set $\operatorname{tok}(\boldsymbol{A})$ of elements called the tokens of $\boldsymbol{A}$,

2. A set $\operatorname{typ}(A)$ of elements called the types of $A$,

3. A family $\left(A_{\alpha}\right)_{\alpha \in \operatorname{typ}(A)}$ of subsets of $\operatorname{tok}(A)$.

For a classification $A$, we say a token $a \in \operatorname{tok}(A)$ has a type $\alpha \in \operatorname{typ}(A), a \models{ }_{A} \alpha$ in symbols, if $a$ belongs to $A_{\alpha}$. We call $\models_{A}$ the classification relation of $A$. We remark that a binary relation $\models$ between two sets $\operatorname{tok}(A)$ and $\operatorname{typ}(\boldsymbol{A})$ gives a classification $A$ such that $A_{\alpha}=\{a \in A \mid a \models=\alpha\}$ and $\models_{A}$ is equal to $\models$. Thus, we have a classification in two ways, giving a family of sets of tokens indexed by each type, or a classification relation between tokens and types. $A_{\alpha}$ is denoted by $\operatorname{tok}_{A}(\alpha)$, and $\operatorname{typ}_{A}(a)$ is defined as a set $\left\{\alpha \in \operatorname{typ}(A) \mid a \models_{A} \alpha\right\}$ of types which the token $a$ has.

A pair $\langle\Gamma, \Delta\rangle$ of subsets of a set $\Sigma$ is called a sequent of $\Sigma$. We say a sequent $\left\langle\Gamma_{1}, \Delta_{1}\right\rangle$ extends a sequent $\left\langle\Gamma_{2}, \Delta_{2}\right\rangle$ and write $\left\langle\Gamma_{2}, \Delta_{2}\right\rangle \leq\left\langle\Gamma_{1}, \Delta_{1}\right\rangle$ if $\Gamma_{2} \subseteq \Gamma_{1}$ and 


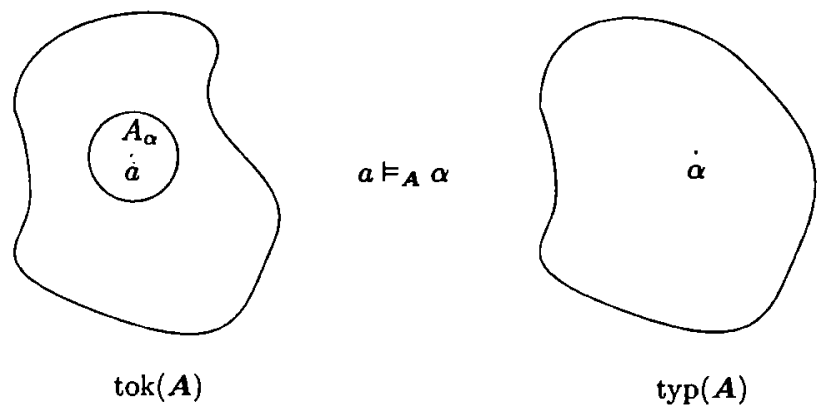

Figure 1: Classification

$\Delta_{2} \subseteq \Delta_{1}$. We say a token $a$ of a classification $A$ realizes a sequent $\langle\Gamma, \Delta\rangle$ of $\operatorname{typ}(A)$ if $\left.\langle\Gamma, \Delta\rangle \leq \operatorname{typ}_{A}(a), \operatorname{typ}(A) \backslash \operatorname{typ}_{A}(a)\right\rangle$, and we say a satisfies $\langle\Gamma, \Delta\rangle$, or $a$ is constrained by $\langle\Gamma, \Delta\rangle$, if $a$ does not realize $\langle\Gamma, \Delta\rangle$. That is, $a$ realizes $\langle\Gamma, \Delta\rangle$ if $a \models_{A}$ $\alpha$ for any $\alpha \in \Gamma$ and $a \nVdash \alpha$ for any $\alpha \in \Delta$, and $a$ satisfies $\langle\Gamma, \Delta\rangle$ if $a$ has some type in $\Delta$ whenever $a$ has all types in $\Gamma$. We also say $a$ is a counter example for $\langle\Gamma, \Delta\rangle$ if $a$ realizes $\langle\Gamma, \Delta\rangle$.

Classifications are structures with which we discuss information. The sets of tokens and types serve a vocabulary, and information is formulated by a binary relation $\models$ and a set of sequents of types. Information flows from one classification to the other along an infomorphism.

Definition 1.2. Let $A_{1}$ and $A_{2}$ be classifications. We say a pair $f=\left\langle f^{\wedge}, f^{\vee}\right\rangle$ of maps $f^{\wedge}: \operatorname{typ}\left(\boldsymbol{A}_{1}\right) \rightarrow \operatorname{typ}\left(\boldsymbol{A}_{2}\right)$ and $f^{\curlyvee}: \operatorname{tok}\left(\boldsymbol{A}_{2}\right) \rightarrow \operatorname{tok}\left(\boldsymbol{A}_{1}\right)$ is an infomorphism from $\boldsymbol{A}_{1}$ to $\boldsymbol{A}_{2}$ and write $f: \boldsymbol{A}_{1} \rightleftarrows \boldsymbol{A}_{2}$ if it satisfies

$$
f^{\wedge}(a) \models{ }_{A_{1}} \alpha \Longleftrightarrow a \models{ }_{A_{2}} f^{\wedge}(\alpha)
$$

for every $\alpha \in \operatorname{typ}\left(A_{1}\right)$ and $a \in \operatorname{tok}\left(A_{2}\right)$.

In the example of chimpanzees, we noticed certain physical states of stones. It is equivalent to considering the classification of the set $A$ of stones by their physical states. Let $\Omega$ be the set of quadruplets of numerical values of volume, weight, solidity and shapes like $\left\langle 10 \mathrm{~cm}^{3}, 5 \mathrm{~g}, 4\right.$, sphere $\rangle$. We consider the classification of $A$ by elements of $\Omega$. That is, if the volume, weight, solidity and shape of $a \in A$ are $10 \mathrm{~cm}^{3}, 5 \mathrm{~g}, 4$ and sphere, $a$ has the type $\left\langle 10 \mathrm{~cm}^{3}, 5 \mathrm{~g}, 4\right.$, sphere $\rangle$. The classification has a typical feature that each element $a$ of $A$ has the unique type. In general, a classification which has such a feature is called a state space.

Definition 1.3. A classification $S=\left\langle S, \Sigma,\left(S_{\sigma}\right)_{\sigma \in \Sigma}\right\rangle$ is called a state space if $\left(S_{\sigma}\right)_{\sigma \in \Sigma}$ is a partition of $S$. For any token $s$ of a state space $S$, the unique type $\sigma$ such that $s \in S_{\sigma}$ is denoted by $\operatorname{state}_{s}(s)$ and called the state of $s$.

Here, we say $\left(S_{\sigma}\right)_{\sigma \in \Sigma}$ is a partition of $S$ if it is a family of subsets of $S$ such that $\cup_{\sigma \in \Sigma} S_{\sigma}=S$ and $S_{\sigma} \cap S_{\tau}=\emptyset$ for each $\sigma \neq \tau$. ( $S_{\sigma}$ can be empty.) A state space is 


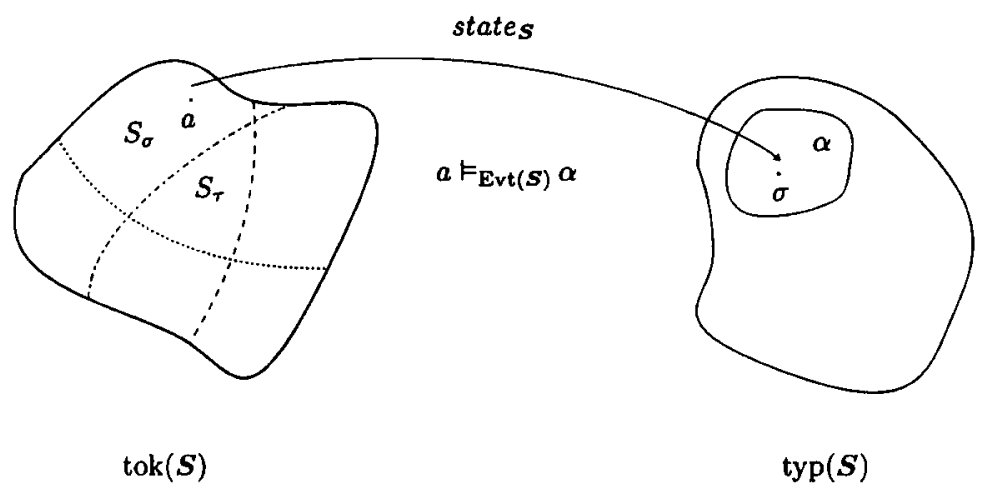

Figure 2: State space

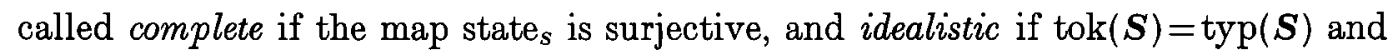
state $_{s}$ is the identity map.

Definition 1.4. We say a sequent $\langle\Gamma, \Delta\rangle$ of $\Sigma$ is a partition if $\Gamma \cap \Delta=\emptyset$ and $\Gamma \cup$ $\Delta=\Sigma$. The free state $\operatorname{space} \operatorname{Ssp}(A)$ associated with a classification $\boldsymbol{A}$ is defined as follows;

1. $\operatorname{tok}(\operatorname{Ssp}(A))=\operatorname{tok}(A)$.

2. $\operatorname{typ}(\operatorname{Ssp}(\boldsymbol{A}))=\{\langle\Gamma, \Delta\rangle \mid\langle\Gamma, \Delta\rangle$ is a partition of typ $(\boldsymbol{A})\}$.

3. state $_{\mathrm{ssp}(A)}(a)=\left\langle\operatorname{typ}_{A}(a), \operatorname{typ}(A) \backslash \operatorname{typ}_{A}(a)\right\rangle$ for $a \in \operatorname{tok}(\operatorname{Ssp}(A))$.

Definition 1.5. The following classification $\operatorname{Evt}(\boldsymbol{S})$ is called the event classification associated with a state space $\boldsymbol{S}$ :

1. $\operatorname{tok}(\operatorname{Evt}(\boldsymbol{S}))=\operatorname{tok}(\boldsymbol{S})$.

2. $\operatorname{typ}(\operatorname{Evt}(\boldsymbol{S}))=\mathscr{F}(\operatorname{typ}(\boldsymbol{S}))$.

3. $\mathrm{s} \models_{\mathrm{Evt}(S)} \alpha$ is defined by $\operatorname{state}_{S}(s) \in \alpha$ for $s \in \operatorname{tok}(\operatorname{Evt}(S))$ and $\alpha \in$ $\operatorname{typ}(\operatorname{Evt}(S))$.

Definition 1.6. Let $S_{1}$ and $S_{2}$ be state spaces. A pair $f=\left\langle f^{\wedge}, f^{\vee}\right\rangle$ of maps $f^{\vee}$ : $\operatorname{typ}\left(\boldsymbol{S}_{1}\right) \rightarrow \operatorname{typ}\left(\boldsymbol{S}_{2}\right)$ and $f^{\sim}: \operatorname{tok}\left(\boldsymbol{S}_{1}\right) \rightarrow \operatorname{tok}\left(\boldsymbol{S}_{2}\right)$ is said to be a (state space) projection from $\boldsymbol{S}_{1}$ to $\boldsymbol{S}_{2}$ and write $f: \boldsymbol{S}_{1} \rightrightarrows \boldsymbol{S}_{2}$ if it satisfies

$$
f^{\wedge}\left(\operatorname{state}_{s_{1}}(a)\right)=\operatorname{state}_{s_{2}}\left(f^{\sim}(\mathrm{a})\right)
$$

for any $a \in \operatorname{tok}\left(\boldsymbol{S}_{1}\right)$.

$A \operatorname{map} f^{\wedge}: \operatorname{typ}\left(\boldsymbol{S}_{1}\right) \rightarrow \operatorname{typ}\left(\boldsymbol{S}_{2}\right)$ induces a map $\left.\left(f^{\wedge}\right)^{-1}: \quad \operatorname{typ}\left(\boldsymbol{S}_{2}\right)\right) \rightarrow \nearrow\left(\operatorname{typ}\left(\boldsymbol{S}_{1}\right)\right)$. We remark that $\left\langle f^{\wedge}, f^{\vee}\right\rangle$ is a projection from $\boldsymbol{S}_{1}$ to $\boldsymbol{S}_{2}$ if and only if $\left\langle\left(f^{\wedge}\right)^{-1}, f^{\vee}\right\rangle$ is an infomorphism from $\operatorname{Evt}\left(\boldsymbol{S}_{2}\right)$ to $\operatorname{Evt}\left(\boldsymbol{S}_{1}\right)$. This infomorphism is denoted by $\operatorname{Evt}(f)$.

\section{Theories}

We remarked in the chimps' example in the previous section that their limited 
knowledge $K$ about the functionality of the stones in $N$ leads to a theory $T(K)$. In general, a theory $T$ over a set $\Sigma$ stands for a systematized information about deductive relations in $\Sigma$. For example, the theory $T(K)$ over the set of functions $\Sigma$ in the chimps' example represents the information about the derivability of functions of breaking nutshells with respect to their limited knowledge $K$. We give a mathematical definition of theories according to [1] and show some of their basic properties.

Definition 2.1. A theory $T$ is a pair $\left\langle\operatorname{typ}(T), \vdash_{T}\right\rangle$ of a set $\operatorname{typ}(T)$ and a binary relation $\vdash_{r}$ on subsets of $\operatorname{typ}(T)$. A sequent $\langle\Gamma, \Delta\rangle$ of $\operatorname{typ}(T)$ is said to be a constraint of $T$ if $\Gamma \vdash_{T} \Delta$, and $T$-consistent if $\Gamma \nvdash_{T} \Delta . \quad T$ is inconsistent if there is no $T$-consistent sequent. We say $T$ is regular if all the constraints of $T$ satisfies the following rules: for any $\alpha$ in $\operatorname{typ}(T)$ and subsets $\Gamma, \Delta, \Sigma^{\prime}$ of $\operatorname{typ}(T)$,

Identity : $\alpha \vdash_{T} \alpha$,

Weakening : $\Gamma, \Gamma^{\prime} \vdash_{T} \Delta, \Delta^{\prime}$ if $\Gamma \vdash{ }_{T} \Delta$,

Global Cut : $\Gamma \vdash_{T} \Delta$ if $\Gamma, \Gamma^{\prime} \vdash_{T} \Delta, \Delta^{\prime}$ for any partition $\left\langle\Gamma^{\prime}, \Delta^{\prime}\right\rangle$ of $\Sigma^{\prime}$.

We say $T$ satisfies Partition rule if every $T$-consistent sequent can be extended to a $T$-consistent partition of $\operatorname{typ}(T)$. It is known that $T$ is regular iff $T$ satisfies Weakening and Partition. (See Proposition 9.9 of [1].) Hence any regular theory $T$ is completely specified by the set of $T$-consistent partitions of $\operatorname{typ}(T)$. That is, Lemma 2.2. Let $\Sigma$ be a set and $K$ be a set of partitions of $\Sigma$. Define a theory $T_{K}=\left\langle\Sigma, \vdash_{K}\right\rangle$ by $\Gamma \vdash_{T} \Delta$ iff $\langle\Gamma, \Delta\rangle \not\left\langle\Gamma^{\prime}, \Delta^{\prime}\right\rangle$ for any $\left\langle\Gamma^{\prime}, \Delta^{\prime}\right\rangle \in K$. Then, $T_{K}$ is a regular theory such that $K$ is the set of $T_{K}$-consistent partitions of $\Sigma$. Conversely, for any regular theory $T$ there exits uniquely a set $K$ of partitions of $\operatorname{typ}(T) \operatorname{such}$ that $T=T_{K}$.

If $\operatorname{typ}(T)$ is a set of functions, then we call $T$ a functional theory. A functional theory should be required to be a regular theory. Let $A$ be a classification. We write $\Gamma \vdash{ }_{A} \Delta$ if every token of $\boldsymbol{A}$ is constrained by $\langle\Gamma, \Delta\rangle$, then $\operatorname{Th}(\boldsymbol{A})=\langle\operatorname{typ}(\boldsymbol{A})$, $\left.\vdash_{A}\right\rangle$ is a regular theory. We call it the theory generated by $A$. The following definition and proposition show that a regular theory induces a classification. See Theorem 9.33 of [1] for a proof of Proposition 2.4.

Definition 2.3. Let $T$ be a regular theory. We define the classification Cla $(T)$ of $T$ as follows :

1. $\operatorname{typ}(\mathrm{Cla}(T))=\operatorname{typ}(T)$,

2. $\operatorname{tok}(\mathrm{Cla}(T))=\{\langle\Gamma, \Delta\rangle \mid\langle\Gamma, \Delta\rangle$ is a $T$-consistent partition of $\operatorname{typ}(T)\}$,

3. $\langle\Gamma, \Delta\rangle-_{\mathrm{Cla}(T)} \alpha$ if and only if $\alpha \in \Gamma$.

Definition 2.4. For any regular theory $T, \operatorname{Th}(\mathrm{Cla}(T))=T$.

The strength of a theory can be measured by the set of constraints. A theory with more constraints is a stronger theory.

Definition 2.5. Let $T_{1}$ and $T_{2}$ be theories with $\operatorname{typ}\left(T_{1}\right)=\operatorname{typ}\left(T_{2}\right)$. We say $T_{1}$ is equal to or weaker than $T_{2}$ and denote $T_{1} \sqsubseteq T_{2}$ if $\Gamma \vdash T_{1} \Delta$ implies $\Gamma \vdash T_{2} \Delta$ for any 
sequent $\langle\Gamma, \Delta\rangle$; that is, any $T_{2}$-consistent sequent is $T_{1}$-consistent.

Proposition 2.6. Let $A$ be a classification and $N \subseteq \operatorname{tok}(A)$. For a sequent $\langle\Gamma, \Delta\rangle$ of $\operatorname{typ}(A)$, define $\Gamma \vdash{ }_{A, N} \Delta$ if and only if every token in $N$ is constrained by $\langle\Gamma, \Delta\rangle$. Let $\operatorname{Th}(\boldsymbol{A}, N)=\left\langle\operatorname{typ}(\boldsymbol{A}), \vdash_{\boldsymbol{A}, N}\right\rangle$. Then,

1. $\operatorname{Th}(A, N)$ is regular.

2. $\operatorname{Th}\left(A, N_{1}\right)\left[\operatorname{Th}\left(A, N_{2}\right)\right.$ if $N_{2} \subseteq N_{1}$.

3. $\operatorname{Th}(A, \emptyset)$ is inconsistent.

4. $\operatorname{Th}(A, \operatorname{tok}(A))=\operatorname{Th}(A)$.

$\mathrm{Th}(\boldsymbol{A})$ is the true theory of a classification $A$. When we regard $N$ as the set of tokens we have at hand, this proposition says that we may expect a stronger theory if we have only fewer tokens in $A$. In the chimps' example, in addition to the fact that they have not checked every stones, they do not have complete information about the checked stones. So the situation is more complicated.

Definition 2.7. Let $\Sigma$ be a set and $K$ be a set of sequents of $\Sigma$.

1. Let $\bar{K}$ be the set of partitions of $\Sigma$ such that $\langle\Gamma, \Delta\rangle \in \bar{K}$ iff $\left\langle\Gamma^{\prime}, \Delta^{\prime}\right\rangle \leq\langle\Gamma$, $\Delta\rangle$ for some $\left\langle\Gamma^{\prime}, \Delta^{\prime}\right\rangle \in K$.

2. Define $\operatorname{Th}(\Sigma, K)$ be the theory $T_{R}$ in Lemma 2.2 .

3. Let $T_{\Sigma}$ be the theory $\operatorname{Th}\left(\Sigma, K_{0}\right)$ where $K_{0}$ is the set of partitions of $\Sigma$.

$K$ stands for a limited knowledge. It is easy to show that $\Gamma \vdash{ }_{K} \Delta$ if and only if $\Gamma \cap \Delta^{\prime} \neq \emptyset$ or $\Gamma^{\prime} \cap \Delta \neq \emptyset$ for every $\left\langle\Gamma^{\prime}, \Delta^{\prime}\right\rangle \in K$ or $\Gamma \cap \Delta \neq \emptyset$, and the theory $T(K)$ in chimps' example is formulated as $\operatorname{Th}(\Sigma, K)$.

Proposition 2.8. Let $\Sigma$ be a set and $K$ be a set of sequents of $\Sigma$.

1. $\operatorname{Th}(\Sigma, K)$ is regular.

2. $\operatorname{Th}\left(\Sigma, K^{\prime}\right) \leftarrow \operatorname{Th}(\Sigma, K)$ if $K \subseteq K^{\prime}$.

3. $\operatorname{Th}(\Sigma, \emptyset)$ is inconsistent.

4. $\operatorname{tok}(\mathrm{Cla}(\operatorname{Th}(\Sigma, K)))=\bar{K}$.

It is usually impossible to obtain the true theory from the limited knowledge. We can regard the limited knowledge $K$ as a set of witnesses which may realize sequents. $\operatorname{Th}(\Sigma, K)$ is the weakest theory such that every $\operatorname{Th}(\Sigma, K)$-consistent sequent has a witness in $K$, and it is one candidate for the true theory we can guess from $K$. It follows from the above proposition that $T_{\Sigma}$ is the weakest regular theory on $\Sigma$.

For a set of functions $\Sigma$, a sequent $\langle\Gamma, \Delta\rangle$ of $\Sigma$ with $\Gamma \cap \Delta=\emptyset$ will be called a specification on $\Sigma$, in the sense that $\langle\Gamma, \Delta\rangle$ specifies an object having any function in $\Gamma$ and no function in $\Delta$. Let $A$ be a classification such that $\operatorname{typ}(A)=\Sigma$. A specification $\langle\Gamma, \Delta\rangle$ on $\Sigma$ is realized by a token $a$ of $A$ if $a=_{A} \alpha$ for every $\alpha \in \Gamma$ and $a \nprec \alpha$ for every $\alpha \in \Delta$, that is, $a$ is not constraind by $\langle\Gamma, \Delta\rangle$. A partition of $\Sigma$ will be called a complete specification. Thus, $\operatorname{tok}\left(\mathrm{Cla}\left(T_{\Sigma}\right)\right)$ is the set of complete specifications, and in general, $\operatorname{tok}(\mathrm{Cla}(T))$ the set of $T$-consistent complete specifications on $\Sigma$ for a regular theory $T$ on $\Sigma$. A complete specification $\langle\Gamma, \Delta\rangle$ 
can be considered as the unique ideal object specified by $\langle\Gamma, \Delta\rangle$ in the sense that $\langle\Gamma$, $\Delta\rangle$ is realized by $\langle\Gamma, \Delta\rangle$ in $\mathrm{Cla}\left(T_{\Sigma}\right)$.

Definition 2.9. Let $S$ be a state space and $\Omega$ be a subset of $\operatorname{typ}(\boldsymbol{S})$. We define a theory $\operatorname{Th}(\boldsymbol{S}, \Omega)=\left\langle y(\operatorname{typ}(\boldsymbol{S})), \vdash^{-} \mathrm{Th}(s, \Omega)\right\rangle$ by

$$
\Gamma \vdash \operatorname{Th}(s, \Omega) \Delta \Longleftrightarrow \Omega \cap \cap \Gamma \subseteq \cup \Delta .
$$

for any sequent $\langle\Gamma, \Delta\rangle$ of $\operatorname{typ}(\operatorname{Evt}(\boldsymbol{S})) . \quad \operatorname{Let} \operatorname{Th}(\boldsymbol{S})=\operatorname{Th}(\boldsymbol{S}, \operatorname{typ}(\boldsymbol{S}))$ and call it the theory associated with $\mathbf{S}$.

Proposition 2.10. Let $S$ be a state space and $\Omega, \Omega^{\prime}$ be subsets of $\operatorname{typ}(S)$.

1. $\operatorname{Th}(S, \Omega)$ is regular.

2. $\operatorname{Th}\left(S, \Omega^{\prime}\right) \sqsubseteq \operatorname{Th}(S, \Omega)$ if $\Omega \subseteq \Omega^{\prime}$.

3. $\operatorname{Th}(S, \emptyset)$ is inconsistent.

4. $\operatorname{Th}\left(S, \Omega_{s}\right)=\operatorname{Th}(\operatorname{Evt}(S))$ for $\Omega_{s}=\{\operatorname{state}(a) \mid a \in \operatorname{tok}(S)\}$.

5. $\operatorname{Th}(\boldsymbol{S})=\operatorname{Th}(\operatorname{Evt}(\boldsymbol{S}))$ if and only if $\boldsymbol{S}$ is complete.

An interpretation defined in the following is a map between theories which moves constraints of one theory to the other. See Definition 9.30 and Exercise 9.15 of [1] for Lemma 2.12 .

Definition 2.11. Let $T_{1}$ and $T_{2}$ be theories. $A \operatorname{map} f: \operatorname{typ}\left(T_{1}\right) \rightarrow \operatorname{typ}\left(T_{2}\right)$ is said to be a theory interpretation from $\operatorname{typ}\left(T_{1}\right)$ to $\operatorname{typ}\left(T_{2}\right)$ if $\Gamma \vdash{ }_{T_{1}} \Delta \operatorname{implies} f[\Gamma] \vdash{ }_{T_{2}} f$ $[\Delta]$ for any sequent $\langle\Gamma, \Delta\rangle$ of $\operatorname{typ}\left(T_{1}\right)$.

Lemma 2.12. Let $A$ and $B$ be classifications.

1. For any infomorphism $\langle\mathrm{f}, \mathrm{g}\rangle$ from $A$ to $B, f: \operatorname{typ}(A) \rightarrow \operatorname{typ}(B)$ is a theory interpretation from $\operatorname{Th}(\boldsymbol{A})$ to $\mathrm{Th}(\boldsymbol{B})$.

2. For any theory interpretation $\mathrm{f}$ from $\operatorname{Th}(A)$ to $\operatorname{Th}(B)$, there exists the unique map $g: \operatorname{tok}(B) \rightarrow \operatorname{tok}(A)$ such that $\langle f, g\rangle$ is an infomorphism from $\boldsymbol{A}$ to $\boldsymbol{B}$.

\section{Functional Schemes}

Now, we shall formulate the latter part of the chimp example in Section 1 as a functional scheme. A functional scheme consists of two classifications, one for the chimps' (or our) imaginary world of functions induced by the theory of functions and another for the state space which stands for the real world, and an infomorphism between them which makes a flow of information between these two classifications. Definition 3.1. A functional scheme is an 8 -tuple $\varsigma=\left\langle\operatorname{tok}(\varsigma), \operatorname{typ}(\varsigma), \vdash\right.$ ๘, $N_{\text {ङ, }}$ $\operatorname{atrb}(\Im)$, state $\left.f_{\Im}, g_{\Im}\right\rangle$ such that

1. $\operatorname{th}(\Im)=\left\langle\operatorname{typ}(\Im), \vdash_{\Im}\right\rangle$ is a regular theory,

2. $\operatorname{st}(\Im)=\left\langle\operatorname{tok}(\Im), \operatorname{atrb}(\Im)\right.$, state $\left._{\varsigma}\right\rangle$ is a state space,

3. $N_{\Im}$ is a subset of tok(ङ),

4. $\left\langle f_{\Xi}, g_{\S}\right\rangle$ is an infomorphism from $\mathrm{Cla}\left(T_{\text {typ(ङ) }}\right)$ to $\operatorname{Evt(st(\Im ))~such~that~}$ 


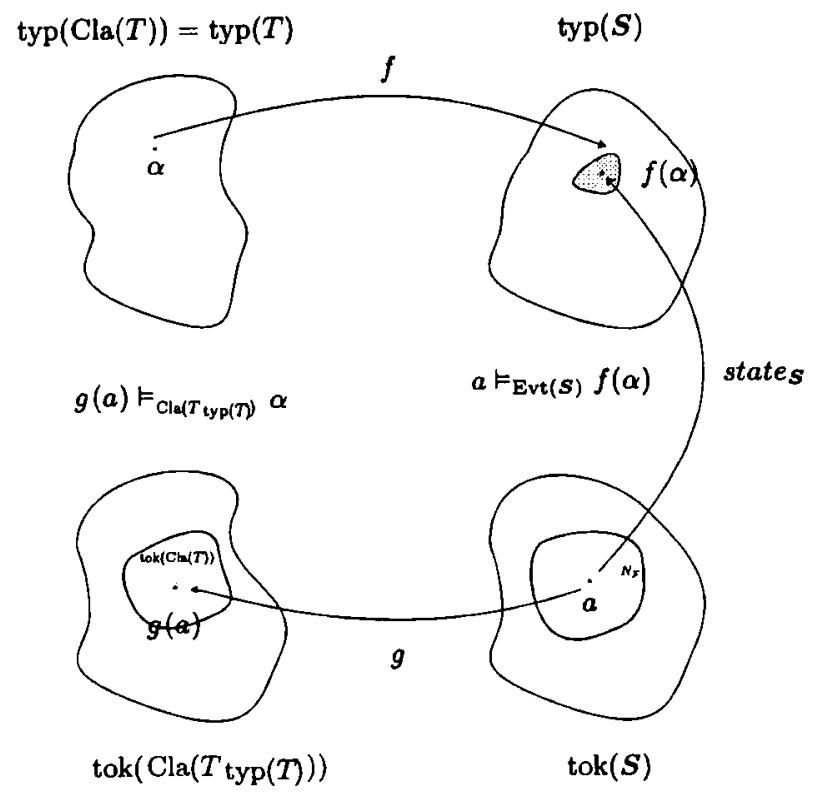

Figure 3: Functional scheme $(T=\operatorname{th}(\mathfrak{S}))$

$$
g_{\S}\left(N_{\Xi}\right) \subseteq \operatorname{tok}(\mathrm{Cla}(\operatorname{th}(\Im)))
$$

th(S) and st(ভ) are called the functional theory and the attribute space of $\subseteq$ respectively. We denote Evt(st(ङ)) by Evt(ङ). An element of $N_{\odot}$ is said to be normal. $f_{\Im}$ and $g_{\S}$ are called the functional interpretation and the functional analysis of $\mathbb{S}$ respectively.

We remark that $f_{\Xi}$ is a map from typ(ङ) to $\boldsymbol{P}(\operatorname{atrb}(\varsigma))$ and $g_{\odot}$ is a map from $\operatorname{tok}\left(\right.$ S) to $\operatorname{tok}\left(\mathrm{Cla}\left(T_{\text {typ(ङ) }}\right)\right)$.

Definition 3.2. A functional scheme $\subseteq$ is said to be complete if $g\left(N_{\subseteq}\right)=$ tok(Cla(th(ङ))) and sound if $N_{\Im}=\operatorname{tok}(\Im)$.

Let us remember the chimps' example. Chimps have the limited knowledge $K=\left\{\left\langle\Gamma_{a}, \Delta_{a}\right\rangle|a \in N\rangle\right.$, and they got a theory $T(K)$ from $K$. Then, they realized that for each function $\alpha$ there is a range $X$ of volume, weight, solidity and shapes such that the state state $(a)$ of a stone $a$ is in $X$ if $a$ has the function $\alpha$. These ranges gave a function $f$ from the set of functions $\Sigma$ to the set of ranges, and also they got a function $g$ such that $g(a)=\langle\{\alpha \in \Sigma \mid$ state $(a) \in f(\alpha)\},\{\alpha \in \Sigma \mid$ state $(a) \notin$ $f(\alpha)\}>$ for a stone $a$. These situations can be formalized as the following proposition.

Proposition 3.3. Let $S$ and $\Sigma$ be a state space and a set, and $g_{N}$ be a map from a subset $N$ of $\operatorname{tok}(\boldsymbol{S})$ to the set sequents of $\Sigma$. Denote $g_{N}(a)$ by $\left\langle\Gamma_{a}, \Delta_{a}\right\rangle$ for every $a \in N$. Assume that for every $\alpha \in \Sigma$ there exists a subset $X$ of $\operatorname{typ}(S) \operatorname{such}$ that

$$
\alpha \in \Gamma_{a} \Longrightarrow \operatorname{state}_{S}(a) \in X
$$




$$
\alpha \in \Delta_{a} \Longrightarrow \operatorname{state}_{S}(a) \notin X
$$

for all $a \in N$. Then, there exists a functional scheme $\subseteq$ such that $\operatorname{st}(\subseteq)=S, N_{\odot}=$ $N, \operatorname{typ}(\Im)=\Sigma$ and $g_{N}(a) \leq g_{\S}(a)$ for all $a \in N$.

Proof. Let $K=\left\{g_{N}(a) \mid a \in N\right\}$ and $\operatorname{th}(\Im)=\mathrm{Th}(\Sigma, K)$. For every $\alpha \in \Sigma$, choose a subset $f_{\Xi}(\alpha)$ of $\operatorname{typ}(S)$ so that $\alpha \in \Gamma_{a}$ implies state $(a) \in f_{\Xi}(\alpha)$ and $\alpha \in \Delta_{a}$ implies state $_{S}(\mathbf{a}) \notin f_{\Xi}(\alpha)$ for all $a \in N$. This can be done by the assumption. Define a map $g_{\tilde{\Sigma}}: \operatorname{tok}(S) \rightarrow \operatorname{tok}\left(\mathrm{Cla}\left(T_{\Sigma}\right)\right)$ so that $g_{\mathbb{\Xi}}(a)=\left\langle\left\{\alpha \in \Sigma \mid a \models_{\operatorname{Evt}(S)} f_{\Sigma}(\alpha)\right\},\left\{\alpha \in \Sigma \mid a \|_{\operatorname{Evt}(S)}\right.\right.$ $\left.\left.f_{\S}(\alpha)\right\}\right\rangle$ for $a \in \operatorname{tok}(S)$. It is clear that $\left\langle f_{\S}, g_{\xi}\right\rangle$ is an infomorphism from $\mathrm{Cla}\left(T_{\Sigma}\right)$ to $\operatorname{Evt}(S)$. It is also clear that $g_{N}(a) \leq g_{\varepsilon}(a)$ for all $a \in N$. Thus, $g_{\approx}[N] \subseteq$ tok(Cla(th(ङ))).

Remark 3.4. Strictly speaking, we have to use the Axiom of Choice of set theory for the choice of $f_{\Xi}$ in the above proof. Design is possible when we can have the function $f$.

Let $\subseteq$ be a functional scheme and $\Omega_{\Xi}=\left\{\operatorname{state}_{\S}(a) \mid a \in N_{\S}\right\}$. Since $\left\langle f_{\S}, g_{\S}\right\rangle$ is an infomorphism from $\mathrm{Cla}\left(T_{\text {typ(ङ) }}\right)$ to $\operatorname{Evt}(\mathrm{st}(\varsigma)), f_{\S}$ is a theory interpretation from

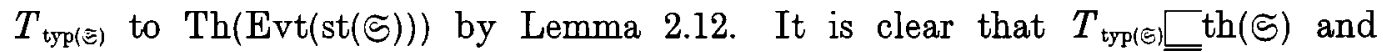

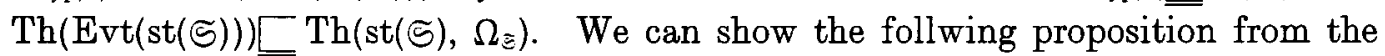
condition $g_{\varepsilon}\left(\overline{N_{\Xi}}\right) \subseteq \operatorname{tok}(\mathrm{Cla}(\operatorname{th}(\varsigma)))$ and Lemma 2.12 .

Proposition 3.5. $f_{\Xi}$ is a theory interpretation from th(ङ) to $\mathrm{Th}\left(\mathrm{st}(\Im), \Omega_{\Xi}\right)$.

The following proposition shows that a functional scheme can be determined by either the functional interpretation or analysis.

Proposition 3.6. Let $T, S, N$ be a regular theory, a state space, a subset of tok $(\boldsymbol{S})$ respectively, and let $\Omega_{x}=\left\{\operatorname{state}_{S}(a) \mid a \in \mathrm{N}\right\}$.

1. For any theory interpretation $f$ from $T$ to $\operatorname{Th}\left(S, \Omega_{N}\right)$, there exists a unique functional scheme such that $T, S, N$ and $f$ are its functional theory, attribute space, normal tokens and functional interpretation, respectively.

2. If a map $g: \operatorname{tok}(S) \rightarrow \operatorname{tok}\left(\mathrm{Cla}\left(T_{\text {typ }(T)}\right)\right)$ satisfies

$$
\forall \alpha \in \Sigma \exists X \subseteq \operatorname{typ}(S) \forall a \in N\left(g(a) \models_{\mathrm{Cla}(T)} \alpha \Longleftrightarrow a \models_{\operatorname{Evt}(S)} X\right),
$$

then there exists a functional scheme such that $T, S, N$ and $g$ are its functional theory, attribute space, normal tokens and functional analysis, respectively.

Proof. 1. Define a map $g: \operatorname{tok}(S) \rightarrow \operatorname{tok}\left(\mathrm{Cla}\left(T_{\mathrm{typ}(T)}\right)\right)$ so that

$$
g(a)=\left\langle\left\{\alpha \in \operatorname{typ}(T) \mid a=_{\operatorname{Evt}(S)} f(\alpha)\right\},\left\{\alpha \in \operatorname{typ}(T) \mid a k_{\operatorname{Evt}(S)} f(\alpha)\right\}\right\rangle
$$

for $a \in \operatorname{tok}(S)$. It is clear that $\langle f, g\rangle$ is an infomorphism from $\mathrm{Cla}\left(T_{\operatorname{typ}(T)}\right)$ to $\operatorname{Evt}(S)$. Let $a$ be a token in $N$. Suppose that $g(a)$ is not $T$-consistent. Put $\left\langle\Gamma_{a}\right.$, $\left.\Delta_{a}\right\rangle=g(a)$. Since $\Gamma_{a} \vdash{ }_{T} \Delta_{a}$ and $f$ is a theory interpretation, we have

$$
\Omega_{N} \cap \bigcap f\left[\Gamma_{a}\right] \subseteq \bigcup f\left[\Delta_{a}\right] .
$$

Since $\operatorname{state}_{\mathcal{S}}(a) \in \Omega_{N} \cap \cap f\left[\Gamma_{a}\right]$, there exists a $\alpha \in \Delta_{a}$ such that $\operatorname{state}_{s}(a) \in f(\alpha)$. 
This is a contradiction. Thus, $g[N] \subseteq \operatorname{tok}(\mathrm{Cla}(T))$, hence we have the desired functional scheme. The uniqueness is clear.

2. The proof of the existence of the functional scheme is similar to that of Proposition 3.3.

Remark 3.7. We can easily deduce that the functional scheme in 2 is unique for each $g$ if $\boldsymbol{S}$ is a complete state space. See also the definition of the similarity of functional schemes which will be given in the end of this section.

Let $\varsigma$ be a functional scheme. We define a binary relation $\models_{\subseteq}$ between tok(ऽ) and $\operatorname{typ}(\Im)$ by $a=_{\varsigma} \alpha$ iff $a \models_{\operatorname{Evt}(\Im)} f_{\Im}(\alpha)$ for $a \in \operatorname{tok}(\Im)$ and $\alpha \in \operatorname{typ}(\Im)$. The classification $\operatorname{cla}(\varsigma)=\left\langle\operatorname{typ}(\Im), \operatorname{tok}(\varsigma), \models_{\epsilon}\right\rangle$ is called the functional classification induced by a functional scheme $\Im$. It is easy to show that $\mathrm{Th}\left(\operatorname{cla}(\Im), N_{\Im}\right)=$ $\operatorname{Th}\left(\right.$ st(ङ), $\Omega_{\odot}$ ), and hence we have th(ङ) $\left[\operatorname{Th}\left(\operatorname{cla}(\subseteq), N_{\odot}\right)\right.$ by Proposition 3.5.

From these observation, we have come to the concept of local logics, which plays the central rôle in Barwise and Seligman [1].

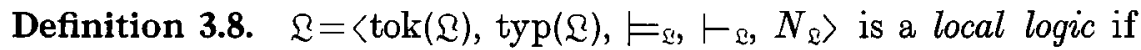

1. $\operatorname{cla}(\mathfrak{L})=\left\langle\operatorname{tok}(\mathfrak{L}), \operatorname{typ}(\mathfrak{L}), \models_{\mathfrak{l}}\right\rangle$ is a classification,

2. $\operatorname{th}(\mathfrak{L})=\left\langle\operatorname{typ}(\mathfrak{R}), \vdash_{\Omega}\right\rangle$ is a regular theory,

3. $\quad N_{\mathfrak{L}}$ is a subset of tok(I) such that $\operatorname{th}(\mathfrak{L})\left[\operatorname{Th}\left(\operatorname{cla}(\mathfrak{L}), N_{\mathfrak{L}}\right)\right.$.

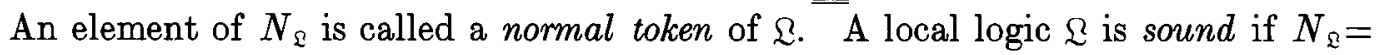
tok $(\mathfrak{L})$ and complete if $\operatorname{th}(\mathfrak{L})=\operatorname{Th}\left(\operatorname{cla}(\mathfrak{L}), N_{\mathfrak{L}}\right)$.

For any functional scheme $\Im,\left\langle\operatorname{tok}(\Im), \operatorname{typ}(\Im), \models_{\odot}, \vdash_{\Im}, N_{\Im}\right\rangle$ is a local logic satisfying the following condition; for every $\alpha \in \operatorname{typ}(\Im)$ there exists a subset $X$ of $\operatorname{atrb}(\varsigma)$ such that $a \models_{\text {cla(ङ) }} \alpha$ iff $a \models_{E_{v t(S)}} X$ for all $a \in \operatorname{tok}(\varsigma)$, a fortiori, for all $a \in$ $N_{\text {s. }}$. Thus we have the following definition.

Definition 3.9. $\mathfrak{L}=\left\langle\operatorname{tok}(\mathfrak{L}), \operatorname{typ}(\mathfrak{L}), \models_{\mathfrak{l}}, \vdash_{\mathfrak{l}}, N_{\mathfrak{l}}\right.$, $\operatorname{atrb}(\mathfrak{L})$, state $\left.{ }_{\mathfrak{l}}\right\rangle$ is a functional logic if

1. $\operatorname{lc}(\Omega)=\left\langle\operatorname{tok}(\Omega), \operatorname{typ}(\Omega), \models_{\Omega}, \vdash_{\mathfrak{l}}, N_{\Omega}\right\rangle$ is a local logic,

2. $\operatorname{st}(\mathfrak{L})=\left\langle\operatorname{tok}(\mathfrak{\Omega}), \operatorname{atrb}(\mathfrak{L})\right.$, state $\left._{\mathfrak{R}}\right\rangle$ is a state space,

3. $\forall \alpha \in \operatorname{typ}(\mathfrak{L}) \exists X \subseteq \operatorname{atrb}(\mathfrak{L}) \forall a \in N_{\mathfrak{L}}\left(a \models_{\text {cla }(\mathfrak{I})} \alpha \Longleftrightarrow a \models_{\operatorname{Evt}(\mathfrak{l})} X\right)$

where $\operatorname{Evt}(\mathfrak{L})=\operatorname{Evt}(\operatorname{st}(\mathfrak{L})) . \quad \operatorname{st}(\mathfrak{L})$ is called the attribute space of $\mathfrak{L}$. A functional logic $\mathfrak{L}$ is sound (complete, resp.) if the local logic lc $(\mathfrak{L})$ is sound (complete, resp.).

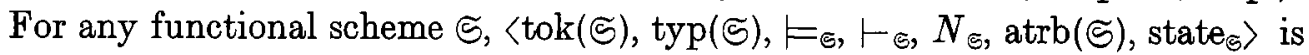
a functinal logic and we denote it by $\log (\subseteq)$. It is clear that the soundness and completeness of $\log (\Im)$ depends on that of the functional scheme $\mathfrak{S}$. Conversely, any functional logic $\mathfrak{L}$ gives a functional scheme $\operatorname{Schm}(\mathfrak{L})$ as follows. Let $\mathfrak{Q}$ be a functional logic. For every $\alpha \in \operatorname{typ}(\mathfrak{L})$ choose a subset $X$ of atrb( $(\mathbb{)}$ such that $a \models_{\text {cla( }(\Omega)} \alpha$ iff $a \models_{\text {Evt( }())} X$ for any $a \in N_{\Omega}$, and put $f_{\mathfrak{\Omega}}(\alpha)=X$. Define a map $g_{\Omega}$ : $\operatorname{tok}(\mathfrak{L}) \rightarrow \operatorname{tok}\left(\mathrm{Cla}\left(T_{\text {typ(\&) }}\right)\right)$ by

$$
\begin{aligned}
& g_{\mathbb{S}}(a)=\left\langle\left\{\alpha \in \operatorname{typ}(\mathfrak{L}) \mid a=_{\operatorname{Evt}(\mathfrak{Q})} f_{\mathcal{L}}(\alpha)\right\},\left\{\alpha \in \operatorname{typ}(\mathfrak{L}) \mid a \nVdash_{\operatorname{Evt}(\mathfrak{I})} f_{\mathcal{R}}(\alpha)\right\}\right\rangle \\
& -120-
\end{aligned}
$$


Let $a$ be an element in $N_{\mathfrak{L}}$ and $\Gamma_{a}=\left\{\alpha \in \operatorname{typ}(\mathfrak{L}) \mid a \models_{\mathrm{cla}(\mathfrak{S})} \alpha\right\}, \Delta_{a}=\{\alpha \in \operatorname{typ}(\mathfrak{L}) \mid a \not \models$ ela(g) $\alpha\}$. Then, $g_{\mathfrak{\Omega}}(a)=\left\langle\Gamma_{a}, \Delta_{a}\right\rangle$. Since $a$ does not satisfy the sequent $\left\langle\Gamma_{a}, \Delta_{a}\right\rangle$ in $\operatorname{cla}(\mathfrak{L}),\left\langle\Gamma_{a}, \Delta_{a}\right\rangle$ is th( $(\mathfrak{)})$-consistent, so $g_{\Omega}(a) \in \operatorname{tok}(\mathrm{Cla}(\operatorname{th}(\Omega)))$. It is clear that $\left\langle f_{\Omega}\right.$, $\left.g_{\Omega}\right\rangle$ is an infomorphism from $\operatorname{Cla}\left(T_{\text {typ(I) }}\right)$ to $\operatorname{Evt}(\Omega)$. Thus we have a functional scheme $\operatorname{Schm}(\mathfrak{L})$ such that $\operatorname{th}(\operatorname{Schm}(\mathfrak{I}))=\operatorname{th}(\mathfrak{I}), \operatorname{st}(\operatorname{Schm}(\mathfrak{I}))=\operatorname{st}(\mathfrak{L}), N_{\operatorname{schm}(\mathfrak{l})}=N_{\mathfrak{l}}$,

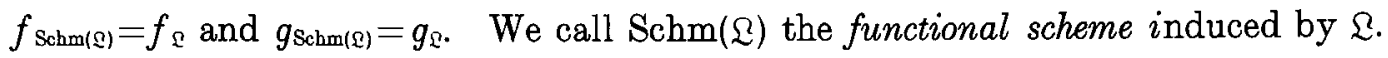

Functional schemes $\mathfrak{S}_{1}$ and $\widetilde{\varsigma}_{2}$ are said to be similar if they have the same functional theory, attribute space, functional analysis, and normal tokens. It is easy to show that two similar functional schemes $\widetilde{S}_{1}$ and $\widetilde{S}_{2}$ satisfy

$$
\forall \alpha \in \operatorname{typ}(T)\left(f_{\Xi_{1}}(\alpha) \cap \Omega_{N}=f_{\Xi_{2}}(\alpha) \cap \Omega_{N}\right)
$$

for $\Omega_{N}=\left\{\operatorname{state}_{\Xi_{1}}(a) \mid a \in N_{\Xi_{1}}\right\} \quad\left(=\left\{\right.\right.$ state $\left.\left._{\Xi_{2}}(a) \mid a \in N_{\Xi_{2}}\right\}\right)$. Similar functional schemes can be identified with each other. Under this identification, the functional scheme obtained in Proposition 3.6 (2) is unique, and also we have the following proposition.

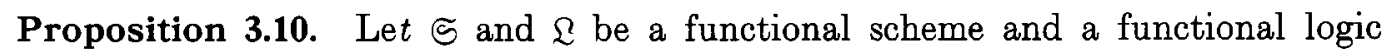
respectively. Then, $\operatorname{Schm}(\log (\Im))=\subseteq$ if $N_{\S}=\operatorname{tok}(\Im)$, and $\log (\operatorname{Schm}(\mathfrak{L}))=\mathfrak{L}$ if $N_{\Omega}=\operatorname{tok}(\Omega)$.

\section{Medium Systems}

We now assume that, instead of being chimps, we as humans are under the same circumstance as in the chimp's example in Section 1. We may break a stone into two pieces if we know that it is too big, and this is a kind of the design activity. We shall try to use our experience and knowledge in a systematic and efficient way so that we get a stone which satisfies our desire. We shall introduce a mathematical system called a medium system in order to formulate our design activity. First, we shall give a review of the situation.

To keep our memory about functions of the stones we tested, we shall make a list in which each entry $d$ is the name of a stone $a$ and the list may contain the features like volume, weight, hardness, shape of $a$, and information about the functions of $a$ like that $a$ has the function $\alpha$ but $a$ does not have the function $\beta$ etc. From the list, we can obtain a state space $D$ such that tok $(D)$ is the set of entries of the list, $\operatorname{typ}(D)$ is the set of quadruplets of the features like $\left\langle 10 \mathrm{~cm}^{3}, 5 \mathrm{~g}, 4\right.$ sphere $\rangle$, and $\operatorname{state}_{D}(d)=\left\langle\sigma_{1}(d), \sigma_{2}(d), \sigma_{3}(d), \sigma_{4}(d)\right\rangle$ where $\sigma_{i}(d)(i=1, \ldots, 4)$ is the content of the list like that $\sigma_{1}(d)$ is the numerical value of the volume of the stone denoted by $d$. $\operatorname{tok}(\boldsymbol{D})$ may contain the names for stones which have not tested if we know their features.

For each name $d \in \operatorname{tok}(D)$ of $a$ stone a, let $\Gamma_{d}$ and $\Delta_{d}$ be the sets of functions $\alpha$ such that it is written in the list that $a$ does have $\alpha$ and $a$ does not have $\alpha$. Then 
$K=\left\{\left\langle\Gamma_{d}, \Delta_{d}\right\rangle \mid d \in \operatorname{tok}(D)\right\}$ is the set of limited knowledge about functions of the stones. From $K$, we can obtain a map $\stackrel{\circ}{e}$ from $\operatorname{tok}(D)$ to the set of sequents on $\Sigma$

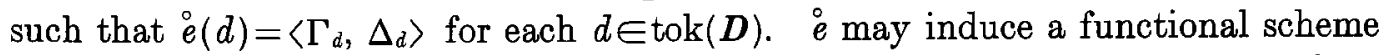
with an infomorphism $e: \operatorname{Cla}\left(T_{\Sigma}\right) \rightleftarrows \operatorname{Evt}(D)$ and a theory $\operatorname{Th}(\Sigma, K)$ such that $\stackrel{\circ}{e}(d)$ $\leq e^{\curlyvee}(d)$ for all $d \in \operatorname{tok}(\boldsymbol{D})$ by Proposition 3.3. Let $\boldsymbol{S}$ be the state space of real stones with $\operatorname{typ}(\boldsymbol{S})=\operatorname{typ}(\boldsymbol{D})$. The correspondence between stones and their names gives a projection $p: \boldsymbol{D} \rightrightarrows \boldsymbol{S}$, where $p^{\smile}(d)$ is the stone denoted by $d$ and $p^{\wedge}$ is the identity map on $\operatorname{typ}(\boldsymbol{D})$. The projection $p: \boldsymbol{D} \rightrightarrows \boldsymbol{S}$ causes an infomorphism $\operatorname{Evt}(p): \operatorname{Evt}(\boldsymbol{S})$ $\rightleftarrows \operatorname{Evt}(\boldsymbol{D})$ as mentioned in Section 1. Let $N \subseteq \operatorname{tok}(\boldsymbol{D})$ be the set of the names of tested stones. It is clear that

$$
\forall \alpha \in \operatorname{typ}(T) \exists X \subseteq \operatorname{typ}(S) \forall d \in N\left(d \models \operatorname{Evt}(\boldsymbol{D}) e^{\wedge}(\alpha) \Longleftrightarrow p^{\smile}(d) \models \operatorname{Evt}(S) X\right)
$$

holds, since $e$ is an infomorphism, $p$ is a projection and $p^{\wedge}$ is identity.

A set $C=\left\{e: \operatorname{Cla}\left(T_{\Sigma}\right) \rightleftarrows \operatorname{Evt}(D), \operatorname{Evt}(p): \operatorname{Evt}(S) \rightleftarrows \operatorname{Evt}(D)\right\}$ of infomorphisms forms a binary channel, a concept introduced by Barwise and Seligman in [1], with its core $\operatorname{Evt}(\boldsymbol{D}) . \quad \mathrm{Cla}\left(T_{\Sigma}\right)$ is a classification which stands for the world of our consciousness, and $\operatorname{Evt}(\boldsymbol{S})$ is for the real world. These two worlds do not have always a trivial correspondence, and it is one of the reason for the difficulties of problem solving. In the channel $C, \mathrm{Cla}\left(T_{\Sigma}\right)$ and $\operatorname{Evt}(S)$ are linked by the core $\operatorname{Evt}(\boldsymbol{D}) . \quad d \in \operatorname{tok}(\boldsymbol{D})$ connects an imaginary (or ideal) stone $e^{\sim}(d) \in \operatorname{tok}\left(\mathrm{Cla}\left(T_{\Sigma}\right)\right)$ specified by the complete $T$-consistent specification

$$
\left\langle\left\{\alpha \in \Sigma \mid d \models_{\operatorname{Evt}(D)} e^{\wedge}(\alpha)\right\},\left\{\alpha \in \Sigma \mid d \nVdash_{\operatorname{Evt}(D)} e^{\wedge}(\alpha)\right\}\right\rangle,
$$

with a real stone $p^{\curlyvee}(d) \in \operatorname{tok}(\operatorname{Evt}(S))$. The rôle of the state space $D$ is that we find a token of $\boldsymbol{D}$ instead of directly finding a token of $\boldsymbol{S}$ when we want to have a token of $\boldsymbol{S}$ satisfying a specification. In this sense, we call $\boldsymbol{D}$ a medium space between a functional theory $T$ and a product space $\boldsymbol{S}$.

Definition 4.1. Let $T$ be a regular theory, and $\boldsymbol{S}$ be a state space. A medium system $D=\langle\boldsymbol{D}, N, e, p\rangle$ between $T$ and $\boldsymbol{S}$ consists of the following:

1. a state space $D$,

2. a subset $N$ of $\operatorname{tok}(\boldsymbol{D})$,

3. an infomorphism $e: \mathrm{Cla}\left(T_{\operatorname{typ}(T)}\right) \rightleftarrows \operatorname{Evt}(D)$,

4. a projection $p: D \rightrightarrows S$

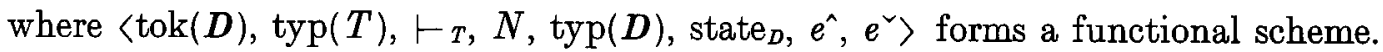
$D$ is called the medium space of $D$.

The necessity of a medium in the framework of General Deisgn Theory was pointed out by Yoshikawa in [7], and it is named a metamodel. (See also Tomiyama and Yoshikawa [5] and Tomiyama et al. [4] for the investigations about metamodels in General Design Theory.) A typical example of medium systems is a production system. In this case, tokens of the medium space are 


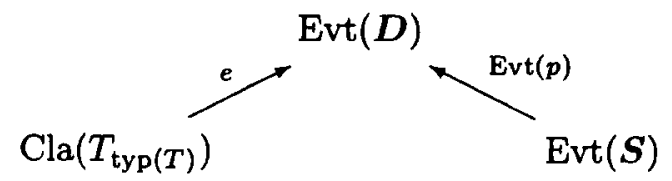

blueprints, and we can call $e^{\wedge}, e^{\curlyvee}$ and $p^{\triangleleft}$ the encoding, the decoding, and the production of $D$ respectively. We can find medium systems in a variety of fields. Programs written in high-level languages, sketches for paintings, and scored music on sheets are tokens of medium space in the medium systems for programming, painting, and music. In the following, we give two examples of medium systems, one is about genetic traits of humans and DNA's and the other comes from formalizations of mathematics.

Example 4.2. We can argue genetic traits of humans and DNA's by a medium system. Let $T$ be the theory of functions of humans and $A$ be a classification of humans by their genetic traits. Then $\operatorname{Ssp}(A)$ is the state space whose tokens are humans and types are the partitions of genetic traits of humans. The world of DNA connects $T$ and $\boldsymbol{A}$. Let $\boldsymbol{D}$ be the state space whose tokens are strands of DNA, whose types are finite strings on $\{A, T, C, G\}$, and state $_{D}(d)$ is the sequence that characterize the strand $d$. A subset $N$ of $\operatorname{tok}(D)$ is defined as the set of strands which actually exist. Define an infomorphism $e: \operatorname{Cla}\left(T_{\text {typ }(T)}\right) \rightleftarrows \operatorname{Evt}(D)$ by $e^{\wedge}(\alpha)$ is the set of finite strings which bring the function $\alpha$ and $e^{\vee}(d)$ is the list of functions which come from the strand $d$. A state space projection $p: D \rightrightarrows \operatorname{Ssp}(A)$ is defined by $p^{\wedge}(\sigma)$ is the partition of genetic traits which indicates that which trait is coded by the string $\delta$, and $p^{\smile}(d)$ is the human who has the strand $d$. Then, $\langle\boldsymbol{D}, N, e, p\rangle$ is a medium system between $T$ and $\operatorname{Ssp}(A)$. This example is a variation of Example 8.16 and 8.23 of Barwise and Seligman [1] .

Example 4.3. Fix a theory (branch) of mathematics (e.g., number theory, group theory, etc.) Let $\operatorname{typ}(T)$ be the set of every (true and false) mathematical assertion in the theory and $\vdash r$ is the set of (known) constraints. In mathematics, what we want to do is to find a proof of a given proposition which is thought to be provable. Define tok $(S)$ be the set of proofs of the theory, $\operatorname{typ}(S)=\operatorname{typ}(T)$, and $\alpha=\operatorname{state}_{S}(a)$ if and only if $a$ is a proof of a proposition $\alpha$. Tis the world of our cognition about mathematics, while $S$ is the objective world of mathematical truth. Formalization of the theory can be regarded as a channel of these two worlds. Fix a first-order language $L$ with which we can do (Hilbert style) formalization of the theory. Let $\boldsymbol{D}$ be a state space such that $\operatorname{typ}(\boldsymbol{D})$ is the set of sentences in $L, \operatorname{tok}(\boldsymbol{D})$ is the set of finite sequences of formulas in $L$, and $\delta=\operatorname{state}_{D}(d)$ if and only if $\delta$ is the last formula of $d$. Let $N$ be a set of formal proofs of propositions. For any $\alpha \in \operatorname{typ}(T)$, $\hat{e}(\alpha)$ is the set of sentences in $L$ which represents the proposition $\alpha$. For any $d \in$ $\operatorname{tok}(D)$, define $E(d)$ be the set of propositions $\alpha$ in $\operatorname{typ}(T)$ such that $d$ is a formal 
proof of $\alpha$, and let $e^{\smile}(d)=\langle E(d), \operatorname{typ}(T) \backslash E(d)$. It is clear that $e$ is an infomorphism from $\operatorname{Cla}\left(T_{\mathrm{typ}(T)}\right)$ to $\operatorname{Evt}(D)$. We can naturally define a state space projection $p$ from $\boldsymbol{D}$ to $\boldsymbol{S}$. Then, $\langle\boldsymbol{D}, N, e, p\rangle$ is a medium system between $\boldsymbol{T}$ and $\boldsymbol{S}$.

Let $\boldsymbol{D}=\langle\boldsymbol{D}, N, e, p\rangle$ be a medium system between $T$ and $\boldsymbol{S}$.

Proposition 4.4. If $p$ satisfies

$$
\forall \alpha \in \operatorname{typ}(T) \exists X \subseteq \operatorname{typ}(S) \forall d \in N\left(d \models_{\operatorname{Evt}(D)} e^{\wedge}(\alpha) \Longleftrightarrow p^{\curlyvee}(d) \models_{\operatorname{Evt}(S)} X\right),
$$

then there are maps $f: \operatorname{typ}(T) \rightarrow P(\operatorname{typ}(S)), g: \operatorname{tok}(S) \rightarrow \operatorname{tok}\left(\operatorname{Cla}\left(T_{\operatorname{typ}(T)}\right)\right)$ and a subset $N^{\prime}$ of $\operatorname{tok}(\boldsymbol{S})$ such that $T, S, N^{\prime}$ and $\langle f, g\rangle$ form a functional scheme. Proof. For every $\alpha \in \operatorname{typ}(T)$ we choose a subset $f(\alpha)$ of $\operatorname{typ}(\boldsymbol{S})$ so that

$$
\forall d \in N\left(d \models=\operatorname{Evt}(D) e^{\wedge}(\alpha) \Longleftrightarrow p^{\curlywedge}(d) \models \operatorname{Evt}(S) f(\alpha)\right) .
$$

Define $g: \operatorname{tok}(S) \rightarrow \operatorname{tok}\left(\mathrm{Cla}\left(T_{\operatorname{typ}(T)}\right)\right)$ so that

$$
g(a)=\left\langle\left\{\alpha \in \operatorname{typ}(T) \mid a \models_{\operatorname{Evt}(S)} f(\alpha)\right\},\{\alpha \in \operatorname{typ}(T) \mid a \nvdash \operatorname{Evt(S)} f(\alpha)\}\right\rangle .
$$

It is clear that $\langle f, g\rangle: \operatorname{Evt}(S) \rightleftarrows \operatorname{Cla}(T \Sigma)$ is an infomorphism. Let $d \in N$ be arbitrary. Then,

$$
g\left(p^{\curlyvee}(d)\right)=\left\langle\left\{\alpha \in \operatorname{typ}(T) \mid d \models_{\operatorname{Evt}(D)} e^{\wedge}(\alpha)\right\},\left\{\alpha \in \operatorname{typ}(T) \mid d \nVdash_{\operatorname{Evt}(D)} e^{\wedge}(\alpha)\right\}\right\rangle .
$$

Therefore, $g\left(p^{\smile}(d)\right)$ is $T$-consistent for any $d \in N$. Thus, $g\left(N^{\prime}\right) \subseteq \mathrm{Cla}(T)$ for $N^{\prime}=$ $\left\{p^{`}(d) \mid d \in N\right\}$. Consequently, $T, S, N^{\prime}$ and $\langle f, g\rangle$ form a functional scheme.

Corollary 4.5. if $\hat{p}^{\wedge}$ is injective and $\mathrm{p}^{\vee}$ is surjective, then there are maps $\mathrm{f}: \operatorname{typ}(T)$ $\rightarrow \boldsymbol{P}(\operatorname{typ}(S)), g: \operatorname{tok}(S) \rightarrow \operatorname{tok}\left(\operatorname{Cla}\left(T_{\mathrm{typ}(T)}\right)\right)$ and a subset $N^{\prime}$ of $\operatorname{tok}(S)$ such that $T$, $S, N^{\prime}$ and $\langle f, g\rangle$ form a functional scheme.

\section{Concluding remarks}

Abstract Design Theory is intended to be a mathematical theory of design. We have proposed the basic notions of the theory and discussed their essential properties in this paper. For the further developments of the theory, we have to (1) consider concrete examples in engineering and other fields in the framework of the theory, (2) analyze mathematical features of the theory, and (3) expand the framework of the theory in order to deal with design processes, circumstances around the artifacts and so on. The analysis of topologies defined in functional schemes has started in Kakuda [2, 3].

\section{Acknowledgements}

This research is partly supported by Japan Society for the Promotion of Science (JSPS) Grants-in-Aid for Scientific Research (No.09440078, No. 12450160, No. 13650068), and the research project "Methodology of Emergent Synthesis" in the 
Research for the Future Program of JSPS. The authors thank Shinzo Kitamura, Kanji Ueda, Toshiharu Taura for stimulative discussions about design. The authors would like to show their gratitude to Takaaki Yagiu for his attention to Abstract Design Theory and valuable comments on former version of this paper.

\section{References}

[1] Jon Barwise and Jerry Seligman, Information Follow, Cambridge Tracts in Theoretical Computer Science 44, Cambridge University Press, 1997.

[2] Yuzuru Kakuda, A mathematical description of GDT-A marriage of Yoshikawa's GDT and Barwise-Seligman's theory of information flow-, Theory GDT'99 Workshop, Cambridge, 1999.

[3] Yuzuru Kakuda, A mathematical definition of synthetic emergence, Proceedings of IWES'99, 13-20, 1999.

[4] Tetsuo Tomiyama, Takashi Kiriyama, Hideki Takeda, Deye Xue and Hiroyuki Yoshikawa, Metamodel : a key to intelligent CAD systems, Research in Engineering Design 1 (1981), 19-34.

[5] Tetsuo Tomiyama and Hiroyuki Yoshikawa, Extended general design theory, Design Theory for CAD, H. Yoshikawa and E.A. Warman (editors), 95-130, Elsevier Science Publishers, 1987.

[6] Shin'ichi Toyoda, Yuzuru Kakuda, Shinzo Kitamura and Tomomi Kotani, Interpreting Abstract Design Theory by simple filter circuit, preprint, 2000.

[7] Hiroyuki Yoshikawa, General design theory and a CAD system, Man-machine Communication in CAD/CAM, T. Sata, E. Warman (editors), 35-57, North-Holland Publishing Company, 1981. 